\title{
Experimental and numerical assessment of shear surface damage using 3D point clouds
}

\author{
S. Karekal CSIRO Earth Science and Resource Engineering, Australia \\ G.V. Poropat CSIRO Earth Science and Resource Engineering, Australia \\ H. Guo CSIRO Earth Science and Resource Engineering, Australia
}

\begin{abstract}
This paper describes modelling of shearing of rock joint surfaces using 3D point clouds generated using photogrammetry. High resolution 3D images of discontinuity surfaces were created before and after shearing. The surface damage due to shearing of joint surfaces was assessed using image processing by comparing the 3D images of undamaged surfaces with those of damaged surfaces. The shear surfaces were concrete replicas of natural surfaces. The damage due to shearing was estimated using numerical modelling in which comparison was made between the concrete surfaces before and after the shear test. It was found that the modelling results were fairly well correlated with the observed surface damage, thus validating the numerical models. The digitised surfaces could also be used to predict anisotropic shear strength and damage of the asperities by numerically shearing the joint surfaces at various directions.
\end{abstract}

\section{Introduction}

The operating depth of open cut mines has increased significantly over the last few years. This has increased the risk associated with pit wall instability and subsequently mining safety. The impact of slope failure in large open pit mines could be very expensive in terms of safety of mine workers, productivity and damage to the machines. There is a need for sustained research and investigation into the optimal excavation design of rock slopes in open pit mines.

A key challenge in managing pit wall stability lies in accurately estimating the shear strength properties of rock discontinuities such as joints, shears, and faults, which is essential for reliable pit wall design and its ongoing management. In situ data collection and parameter estimation has been limited to date by safety restrictions and physical measurement limitations.

The roughness of a discontinuity has an important influence on the shear strength of a discontinuity. Therefore characterisation of surface irregularities and conditions and assessment of surface damage during deformation is important in the analysis of the stability of rock slopes. Determining the roughness of a discontinuity surface and providing a quantitative measure of the roughness would require considerable effort and often cannot be done because of safety issues inherent in conventional techniques that require direct contact with the rock surface.

This paper describes modelling and assessment of shear surface damage using 3D point clouds.

\section{3D point clouds}

For any asperity damage modelling due to shear, one must have 3D digitised surfaces of the joint/discontinuities. This representation of a joint surface in 3D can be made using point clouds. Point clouds are a series of $X, Y, Z$ coordinates. Laser and photogrammetry systems can produce clouds of 3D points. The resolution and range accuracy associated with the acquisition of 3D points which are capable of producing digital surface model representing the topography of the surfaces of joint/discontinuity are important. Such point clouds are generally irregularly spaced in 3D space unless very specific geometrical constraints apply and such constraints almost always do not apply in field measurements. Data acquisition 
must have sufficient number of measurements so that a usable model of the surface topography can be created. The acquisition and use of point cloud data should be undertaken with due consideration of the accuracy, resolution and precision of the data. Once the realistic estimate of the surface morphology is modelled, a range of techniques can then be applied for surface characterisation.

In general, depending upon the applications, the point clouds must be transformed to an appropriate data structure or spatial basis to ensure that the data is in the format required to model surface damage due to shear. The use of this transformation has been well documented in the literature (Baker et al., 2008; Haneberg, 2007). A typical 3D image of a part of an exposed surface is shown in Figure 1 and the corresponding point cloud is shown in Figure 2. The point cloud consists of 660,000 spatial data points on 300 micron spacing.
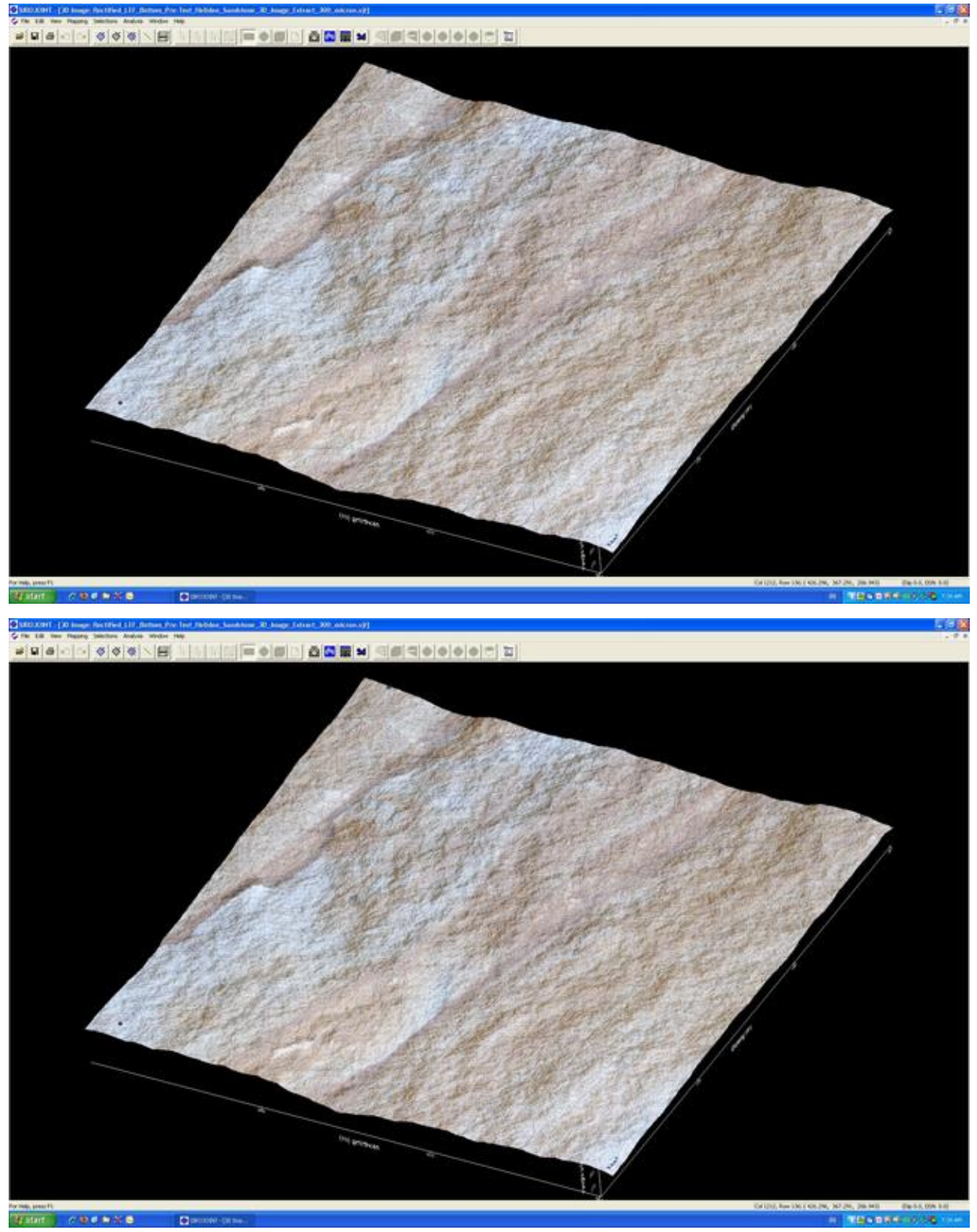

Figure $13 \mathrm{D}$ image of a sandstone surface 

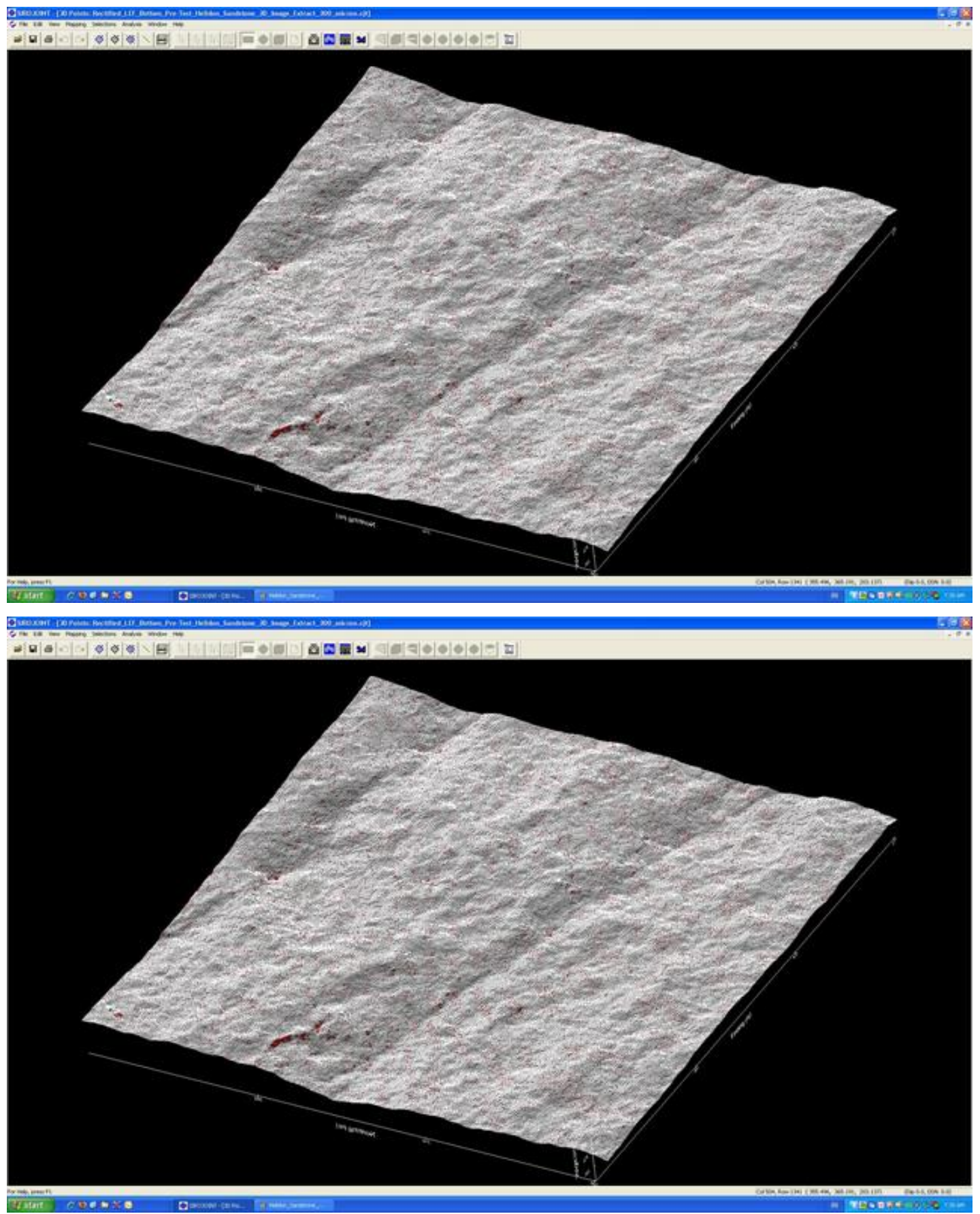

Figure 2 Point cloud representation of sandstone surface

\subsection{Measurement noise and errors}

The principal measurement error that must be addressed is the error in measuring the range from the sensor to a point on an exposed discontinuity. Range measurement noise is generally dominated by the resolution and precision of the measurement system but may be significantly increased by other effects.

The effect of measurement errors is, in most cases, to increase the apparent roughness of a surface. Range errors can be reduced with the application of some data processing techniques. One easily implemented technique is range averaging. It should be noted that the application of data processing may change the statistical distribution of the errors and this change may affect the choice of any preconditioning applied to the range data before roughness is estimated. For example, simple range averaging over a patch (for example low pass filtering with a rectangular uniformly weighted window) will change the error distribution 
due to resolution limits from uniformly distributed over a range defined by the resolution interval to a distribution that may approach that of a Gaussian distributed random variable if a sufficiently large number of samples is acquired.

\section{$3 \quad$ Laboratory shear tests using concrete surface replicas}

In general, it is quite difficult to collect a sufficient number of samples of surfaces of exposed rock discontinuities and even harder to find rock samples with identical surface roughness for repeated shear tests at different normal stresses and shear directions required for this study.

In view of these difficulties, it was decided to make concrete replicas of a natural joint surface and carry out tests on these artificial surfaces to investigate the effect of normal stresses, and shear direction. Polyurethane moulds were prepared which had imprints of the naturally broken joint surfaces. These moulds were then used to cast concrete blocks containing joint surface replicas for shear testing. The UCS of the concrete block was $40 \mathrm{MPa}$ after curing for over 28 days. The joint surface strength was also of the same order (40 MPa). The dimensions of these concrete joint surfaces were 270 by $270 \mathrm{~mm}$ to fit in the custom made shear box (Figure 3).
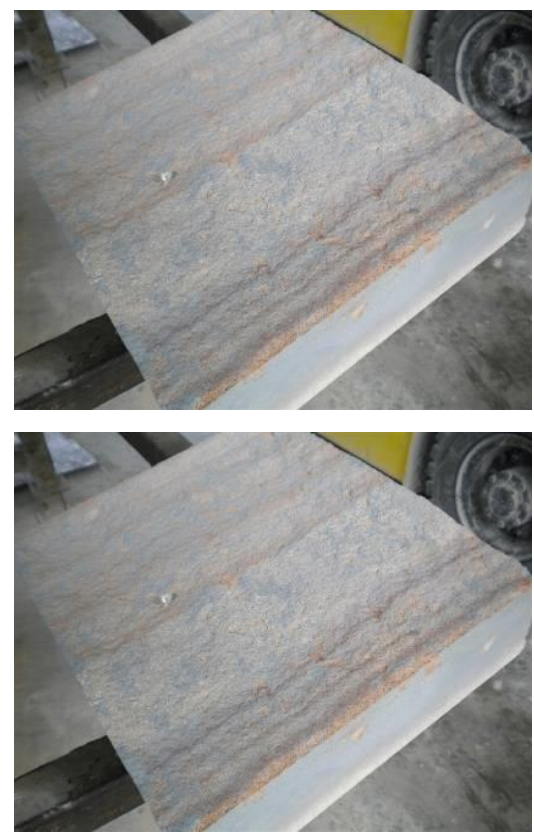

(a)
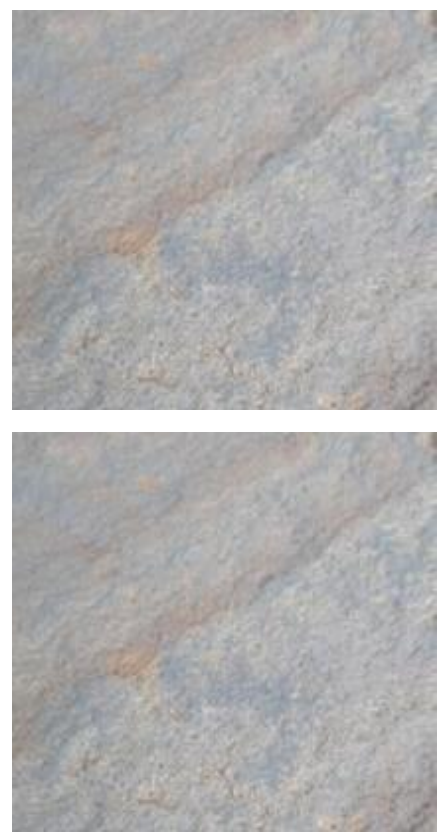

(b)
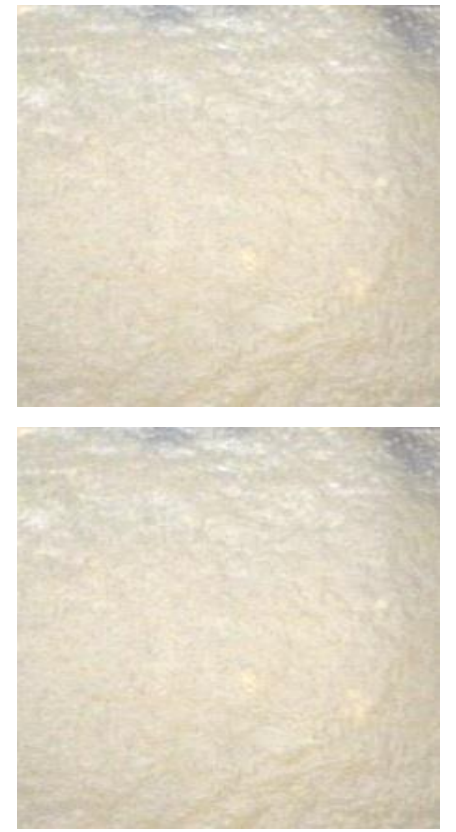

(c)

Figure 3 Surface of original sandstone sample surface (a); detail of the rock surface (b); and the Polyurethane mould of the surface to $270 \times 270 \mathrm{~mm}$ size (c)

The block was sheared at a constant rate of $0.266 \mathrm{~mm} / \mathrm{min}$. Normal stress of $2.72 \mathrm{MPa}$ was applied on the concrete joint specimen. To assess the reproducibility of the shear tests, two concrete surfaces were tested under the same the conditions at $2.72 \mathrm{MPa}$ normal stress. The results of these tests, that is, the behaviour shear stress and shear displacement of concrete blocks under that normal stress is shown in Figure 4. Except for initial deformation variation, the two shear samples behaved almost identically with very slight peak variations. JRC of the concrete joint surface is about 13. 


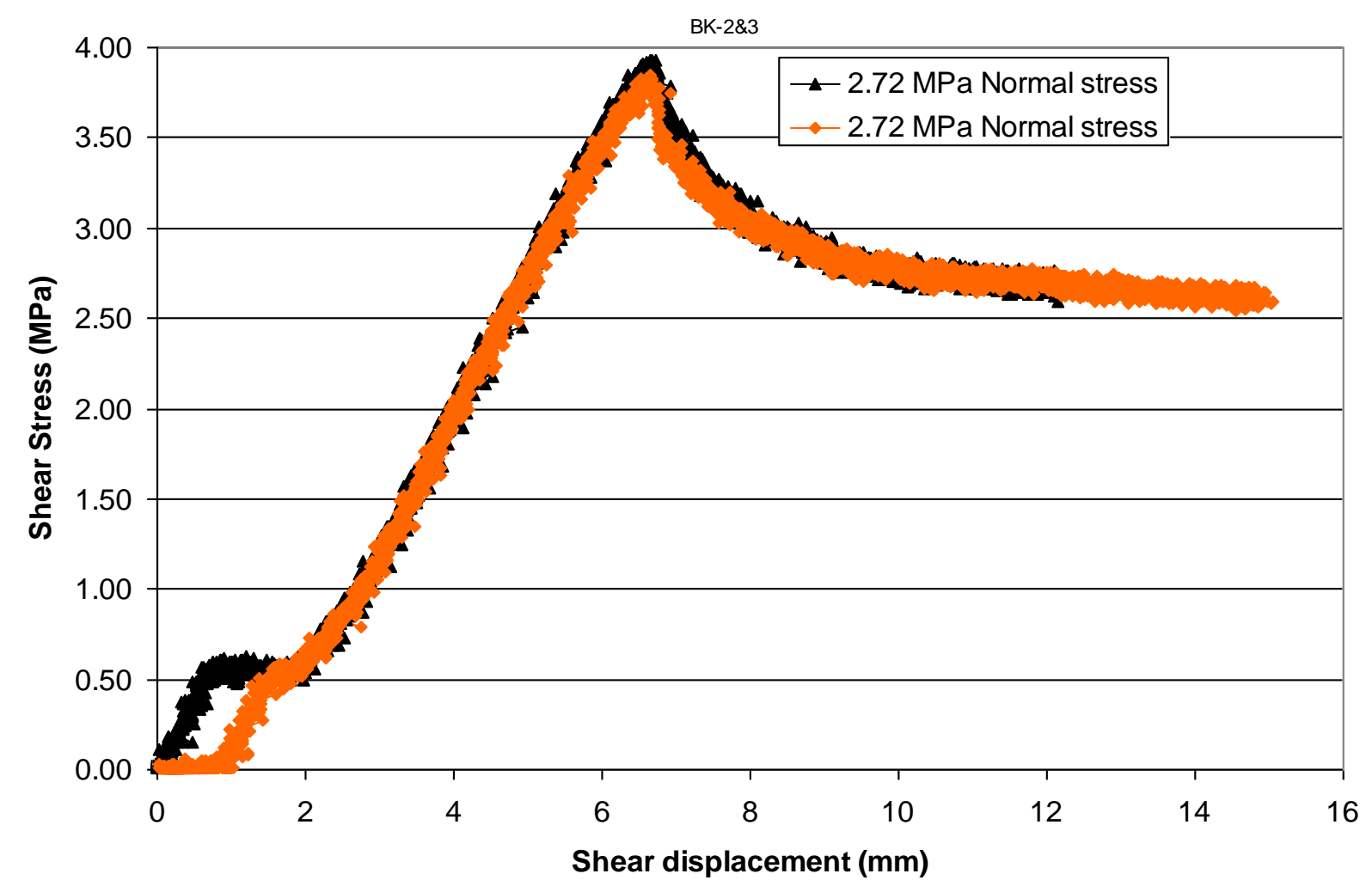

Figure 4 Shear stress versus shear displacement reproducibility test results under $2.72 \mathrm{MPa}$ normal stress

\subsection{Registration of 3D surface images}

Three-dimensional surface images of the surface were captured before and after testing at resolutions of one pixel per spatial data point and three pixels per spatial data point to examine the surface damage in detail. Rigorous checking was performed to identify occlusions in the 3D data arising from mismatched data points.

The scale of the 3D images was controlled by the use of four control points located on the corners of the shear boxes as shown in Figure 5 . The locations of these control points in the local reference frame of the shear box were accurately determined, and the positions and orientations of the shear box relative to the cameras were determined using standard photogrammetric techniques.

\section{$4 \quad$ Surface damage assessment}

The assessment of surface damage was carried out by two methods. One method is based on numerical modelling of digitised surface model by adopting the procedure given by Huang et al. (2002). The second method is an experimental method based on registering the 3D images of the replica and rock surfaces before and after the shear test, and the surface damage is quantified using pattern matching algorithm of these images.

In numerical modelling of fractures with rock joint asperities, Huang et al. (2002) gave a procedure for modelling using Mohr-Coulomb failure criterion. This procedure was used for large scale computation to model the surface damage due to shearing the joint surfaces under a given normal stress. The details can be found in a report by Guo et al. (2011). A digitised 3D surface as a series of X, Y, Z coordinates was used for numerical modelling. $X$ and $Y$ define a regular grid on the average plane of the discontinuity and $Z$ represents the distance from the average plane. The nature of the surface and the data acquisition process (camera position) make re-sampling to a regular XY grid straightforward and accurate enough for the purpose of the analysis. The rough shear surface is triangulated to form a continuous surface for numerical modelling of shearing of surface asperities. 
Figure 6 shows a comparison among unsheared surface (Figure 6(a)), visually observed surface damage (Figure 6(b)) after shearing and predicted surface damage (Figure 6(c)) from numerical modelling at $2.72 \mathrm{MPa}$ normal stress on the 270 by $270 \mathrm{~mm}$ concrete surface after about $15 \mathrm{~mm}$ shear displacement. The surface damage predicted from the numerical modelling is found to match well with the observed damage at most locations on the joint surface, thus validating the results of the numerical simulation.

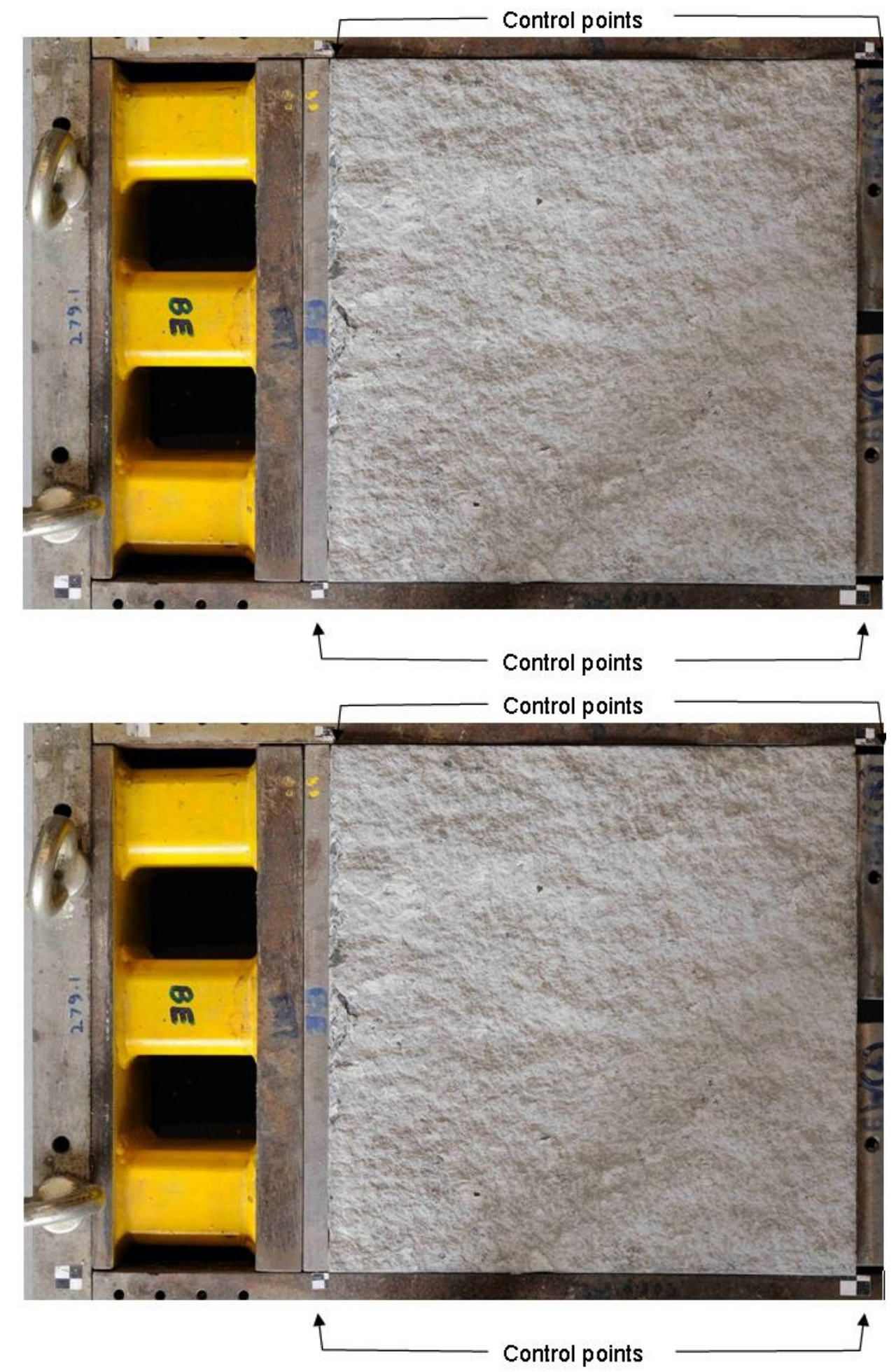

Figure 5 Bottom shear box with targets used for control points. The dimensions of the concrete block are $270 \mathrm{~mm}$ length, $270 \mathrm{~mm}$ width and $135 \mathrm{~mm}$ depth 


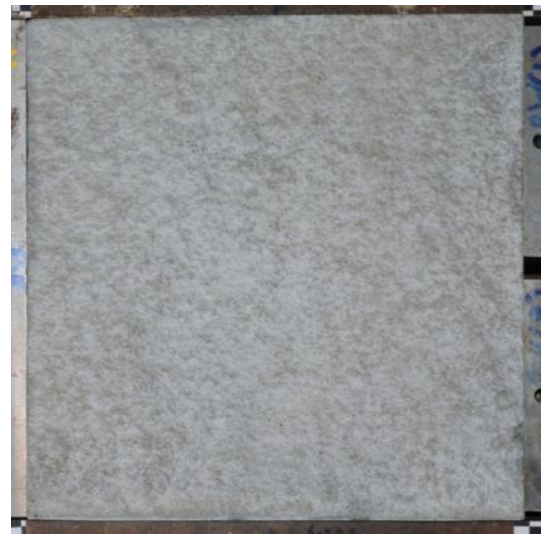

(a)

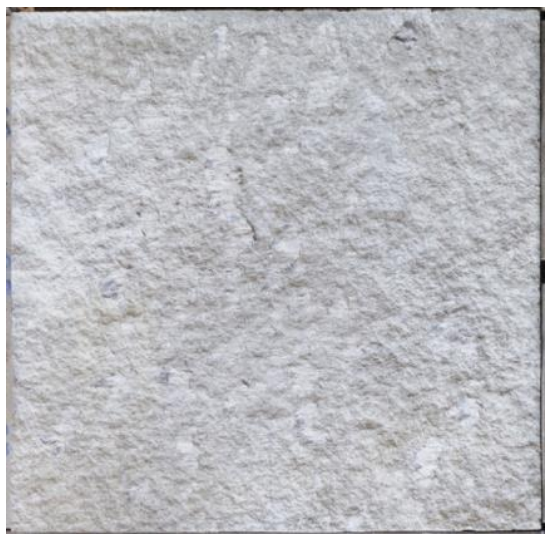

(b)

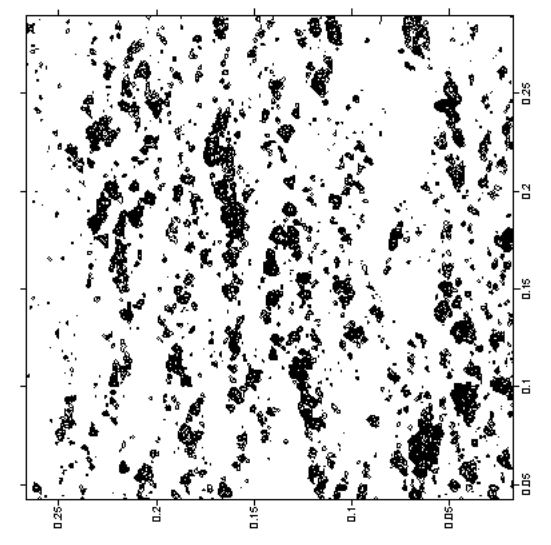

(c)

Figure 6 Comparison of surface damage at 2.72 MPa applied normal stress; unsheared surface (a); visually observed sheared surface damage (b); numerically predicted surface damage (c). Shear direction is from left to right, and shear travel is about $15 \mathrm{~mm}$

In the second method, the assessment of surface damage was performed on a 270 by $270 \mathrm{~mm}$ concrete sample by registering the 3D images of the replica and rock surfaces before and after the shear test. This was performed using the control points established on the shear box. The details can be found in a report by Guo et al. (2011). The surface damage is quantified based on pattern matching algorithm that compares the photographs of undamaged surface (before the shearing the joints, Figure 7(a)) with that of damaged surface (after shearing, Figure $7(b))$. The damage locations observed visually on the concrete surface (Figure $7(b)$ ) matched well with the damage recognised by the pattern matching algorithm (Figure $7(c)$ ).

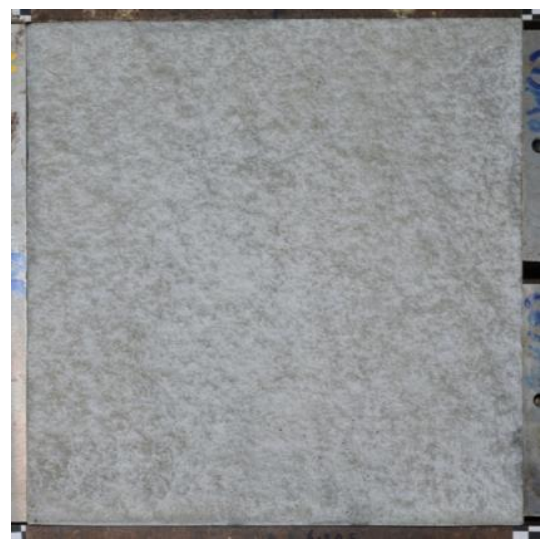

(a)

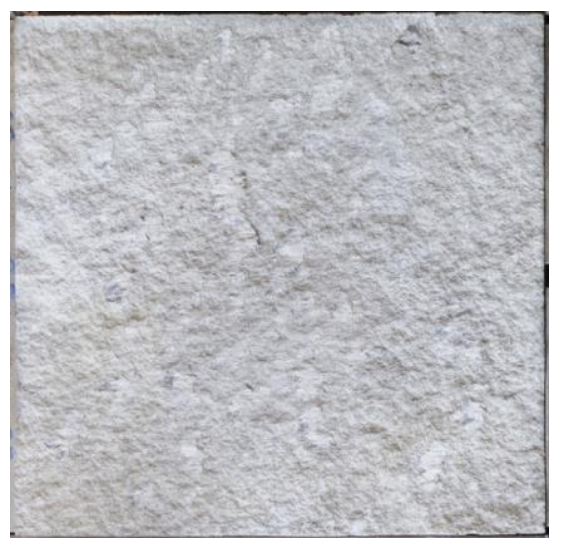

(b)

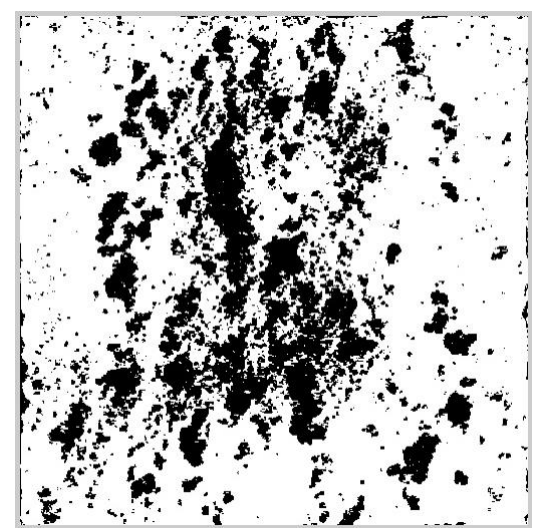

(c)

Figure 7 Comparison of surface damage at 2.72 MPa applied normal stress; unsheared surface (a); visually observed sheared surface damage (b); measured surface damage by pattern matching algorithm (c). Shear direction is from left to right, and shear travel is about $15 \mathrm{~mm}$

\section{$5 \quad$ Workflow for 3D image generation of exposed joint surfaces}

The workflow required for the creation of a 3D image or images is described in Figure 8. The workflow described is specific to the use of Sirovision but is broadly similar to the use of any image based 3D imaging system that produces dense point clouds suitable for estimation of the detailed structure of a surface and thus the JRC and shear strength.

The detail of these steps will vary depending on the operational requirements however the key requirement is to obtain overlapping images with sufficient resolution to meet the measurement 
requirements and with the camera geometry being such that the automated processing works reliably and the measurement precision meets the task requirements.

Creating a Geo-referenced 3D Image

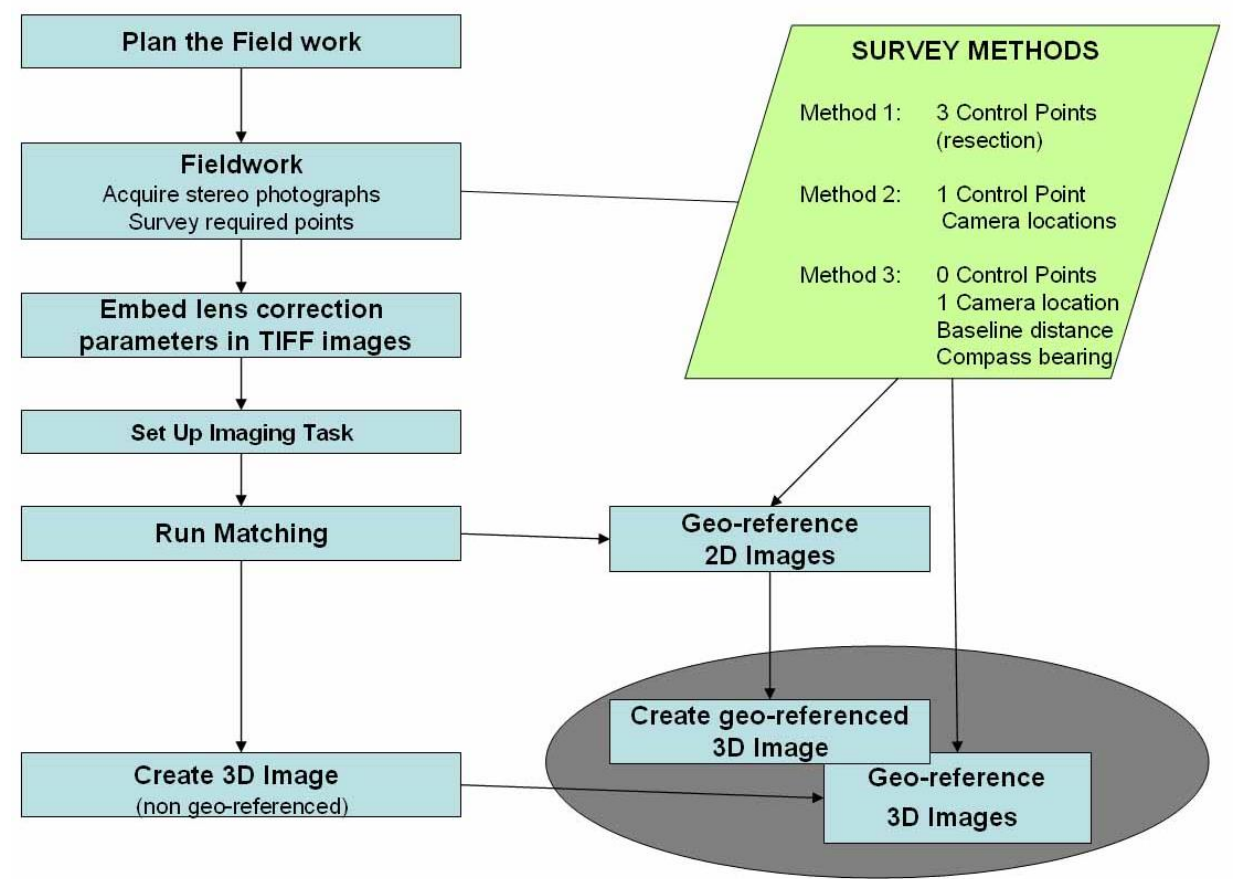

Figure 8 Top level description of the work flow for 3D image generation

\section{Conclusions}

A technique has been developed to remotely map the rock joints/discontinuities generating a high resolution 3D model of the surface at millimetre resolution to determination of the roughness of the exposed discontinuities and support accurate modelling of discontinuity behaviour under shearing. The technique was based on the use of 3D imaging developed by CSIRO which was successfully used to obtain 3D surface models of concrete blocks that replicated the surface roughness of discontinuities in sandstone sample. A pattern correlation algorithm was used to assess the surface damage to the asperities by comparing the differences between the undamaged surface and damaged surface. Further, the modelled surface damage correlated reasonably well with the surface damage observed experimentally on the concrete surface thus validating the numerical models. Such a procedure can be adopted to estimate the shear strength and surface damage of discontinuities in the field based on the 3D point cloud data collected remotely on the exposed joint surfaces in open pit mines.

\section{Acknowledgement}

ACARP is gratefully acknowledged for funding a part of this research program. We also thank Phillip Soole for taking photographs of concrete surfaces and processing the data and Cedric Lambert for providing his basic code.

\section{References}

Baker, B.R., Gessner, K., Holden, E. and Squelch, A. (2008) Automatic detection of anisotropic features on rock surfaces, Geosphere, Vol. 4, No. 2, pp. 418-428.

Haneberg, W.C. (2007) Directional roughness profiles from three-dimensional photogrammetric or laser scanner cloud points, in Proceedings 1st Canada-US rock mechanics symposium, E. Eberhardt, D. Stead and T. Morrison (eds), 27-31 May 2007, Vancouver, Canada, pp. 101-106.

Huang, T.H., Chang, C.S. and Chao, C.Y. (2002) Experimental and mathematical modelling for fracture of rock joint with regular asperities, Engineering Fracture Mechanics, Vol. 69, pp. 1977-1996. 
Guo, H., Karekal, S., Poropat, G. and Soole, P. and Lambert, C. (2011) Pit wall strength estimation with 3D imaging, ACARP Project Number C18030, Australian Coal Association Research Project Publication, 103 p. 